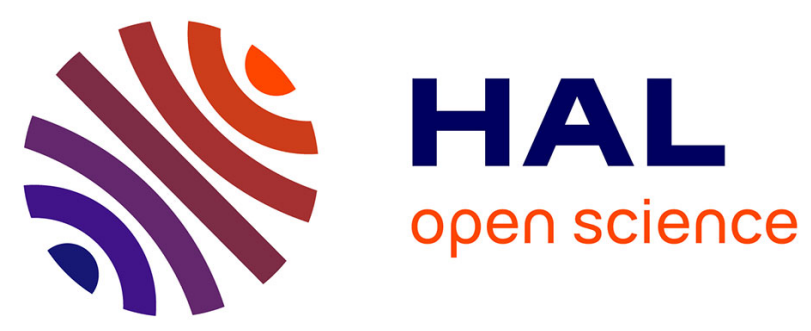

\title{
Mesoscale modeling and experimental analyses for pantographic cells: effect of hinge deformation
}

\author{
Mario Spagnuolo, Ugo Andreaus, Anil Misra, Ivan Giorgio, François Hild
}

\section{To cite this version:}

Mario Spagnuolo, Ugo Andreaus, Anil Misra, Ivan Giorgio, François Hild. Mesoscale modeling and experimental analyses for pantographic cells: effect of hinge deformation. Mechanics of Materials, 2021, 160, pp.103924. 10.1016/j.mechmat.2021.103924 . hal-03230384

\section{HAL Id: hal-03230384 \\ https://hal.science/hal-03230384}

Submitted on 19 May 2021

HAL is a multi-disciplinary open access archive for the deposit and dissemination of scientific research documents, whether they are published or not. The documents may come from teaching and research institutions in France or abroad, or from public or private research centers.
L'archive ouverte pluridisciplinaire HAL, est destinée au dépôt et à la diffusion de documents scientifiques de niveau recherche, publiés ou non, émanant des établissements d'enseignement et de recherche français ou étrangers, des laboratoires publics ou privés. 


\title{
Mesoscale modeling and experimental analyses for pantographic cells: effect of hinge deformation
}

\author{
Mario Spagnuolo $^{\mathrm{a}, \mathrm{b}, *}$, Ugo Andreaus $^{\mathrm{c}}$, Anil Misra $^{\mathrm{d}}$, Ivan Giorgio ${ }^{\mathrm{e}, \mathrm{b}}$, François Hild $^{\mathrm{f}}$ \\ ${ }^{a}$ Dipartimento di Ingegneria Civile, Ambientale e Architettura (DICAAR), Università degli Studi di Cagliari, Cagliari, Italy \\ ${ }^{b}$ International Research Center for the Mathematics and Mechanics of Complex Systems, University of L'Aquila, Italy \\ ${ }^{c}$ Università degli Studi di Roma "La Sapienza", DISG, Italy \\ ${ }^{d}$ Civil, Environmental and Architectural Engineering Department, University of Kansas, 1530 W. 15 th Street, Learned Hall, Lawrence, KS \\ 66045-7609, USA \\ ${ }^{e}$ Department of Civil, Construction-Architectural and Environmental Engineering, University of L'Aquila, Italy \\ ${ }^{f}$ Université Paris-Saclay, ENS Paris-Saclay, CNRS \\ LMT - Laboratoire de Mécanique et Technologie, Gif-sur-Yvette, France
}

\begin{abstract}
In order to synthesize exotic metamaterials, it was proposed to use as elementary components of metamaterial microstructures so-called pantographic cells. The use of a mesomodel based on Euler-Bernoulli nonlinear beam theory is utilized for describing their behavior when their characteristic sizes are of the order of millimeters. With an in-house code, in which the connecting hinges were modeled as elastic extensional and torsional elements, simulations were performed and compared with experimental data. The comparison was carried out for compression and extension tests. It is proven, both numerically and experimentally, that the hinges undergo different deformation mechanisms. As a consequence, for compression and extension tests, the distribution and the total value of the strain energy vary significantly. The analysis is made possible in parts via Digital Image Correlation, which allows kinematic fields to be measured and the hypotheses employed for developing the proposed model to be probed experimentally.
\end{abstract}

Keywords: Digital Image Correlation, Euler-Bernoulli beam model, Extensible metamaterials, Large microstructural torsion, Pantographic cells

\section{Introduction}

In the history of Science, the construction of models ${ }^{23}$ describing physical phenomena has been triggered by the ${ }_{25}$ actual feasibility of generating experimental proofs. In ac- ${ }_{26}$ cordance with this approach, the Cauchy version of Con- ${ }_{27}$ tinuum Mechanics has seen a successful amount of valida- ${ }_{28}$ tions. Classic Continuum Mechanics assumes some ad hoc ${ }_{29}$ limitations in the selection of the terms to be used in the ${ }_{30}$ energy of deformable media [11. These hypotheses were ${ }_{31}$ generalized to obtain new mechanical models. They are ${ }_{32}$ reasonable when looking for simple models that lead to $\mathrm{a}_{33}$ wide domain of validity. As every mathematical model, ${ }_{34}$ Cauchy Continuum Mechanics has a limited range of ap- ${ }_{35}$ plicability. In the present paper, the mechanical behavior of pantographic cells is investigated as they constitute ${ }_{37}$ an important microstructural element of second gradient ${ }_{38}$ metamaterials 1 .

As an example of aforementioned limitations, it was established for Cauchy continua that the macroscopic strain ${ }_{41}$ energy a priori includes only the first displacement gradients. This choice may be accepted without any doubt ${ }_{43}$

* Corresponding author

Email address: mario.spagnuolo@unica.it (Mario Spagnuolo) ${ }^{46}$ on the basis of experimental evidences for many materials. However, it becomes too restrictive when modeling different, tailored-designed materials, e.g. for so-called metamaterials 3]). In the present work, it is proven that, when using pantographic cells as metamaterial microstructure, it is necessary, after homogenization, to use generalized micro-stretched continua. In particular, when the microdeformability of connecting hinges is not negligible, it may be appropriate to specify the kinematics of introduced generalized continua with two placement fields [26]. It is noteworthy that such micro mechanisms play significant role in the deformation of natural as well as synthesized granular materials [17.

In the classic works [16, 21, the authors have demonstrated that the presence of a microstructure can be taken into account from the macroscopic viewpoint by also considering (at least) second gradients of displacements in the strain energy of the microstructured (architectured) material. This observation has led to the development of various constitutive postulates [14, 15].

Pantographic structures are particular metamaterials that can be modeled by means of such second gradient continua [12, 24]. The underlying microstructure (sometimes simply called pantographic structure) consists of a grid made up of two layers of parallel fibers (or beams) in- 
terconnected by perfect hinges (free rotations) or deforming hinges (with torsional stiffness), which are sometimes referred to as pivots. The pantographic metamaterial is normally studied by means of two types of models, one discrete, based on a system of springs, and one continuum, obtained from the discrete one by a suitable homogenization procedure, as it is shown in [12. The model we use in this work is a mesoscale model in the sense that it models each fibre as a continuum medium (a non-linear EulerBernoulli beam) and therefore this model is in a middle position between the two above mentioned. However, for the hinges we chose to consider a model that is in effect discrete because it models the torsion deformation with a rotational spring and the pivot shear deformation with an extensional spring. On the other hand, modeling pivots with a beam element does not seem appropriate. In this sense, the model presented in this paper is multiscale.

In literature many examples of similar architectured metamaterials are present. In [10] the authors study the effects of the architecture of mechanical metamaterials on their mechanical properties, showing experimental examples and numerical simulations. In 30, on the other hand, the authors study the deformations of stretchable metabeams. The cited examples provide, from a model point of view, first gradient continua. The case of the metamaterial studied in this paper is slightly different. As we have already remarked, the pantographic metamaterial has been specifically designed to give rise, after homogenization, to a second gradient continuum model. The model based on 91 the non-linear Euler-Bernoulli beam model is limited to 92 the study of cases where there are few fibers and these are 93 arranged in such a way as to obtain a low-density lattice. ${ }_{94}$ Furthermore, in this paper a study is conducted to directly 95 model the deformation of individual hinges. The informa- 96 tion obtained by studying this case with only three cells 97 will be important later for the study of denser architec- 98 tures, where the mechanical response of the single hinge is 99 difficult to separate from that of the rest of the elementary 100 components.

The minimal pantographic structure that can be de-102 signed (and that preserves the basic mechanical features 103 of this metamaterial) consists of a three-element panto-104 graph, namely a structure that is globally similar to that ${ }_{105}$ of three successive crosses (Figure 1).

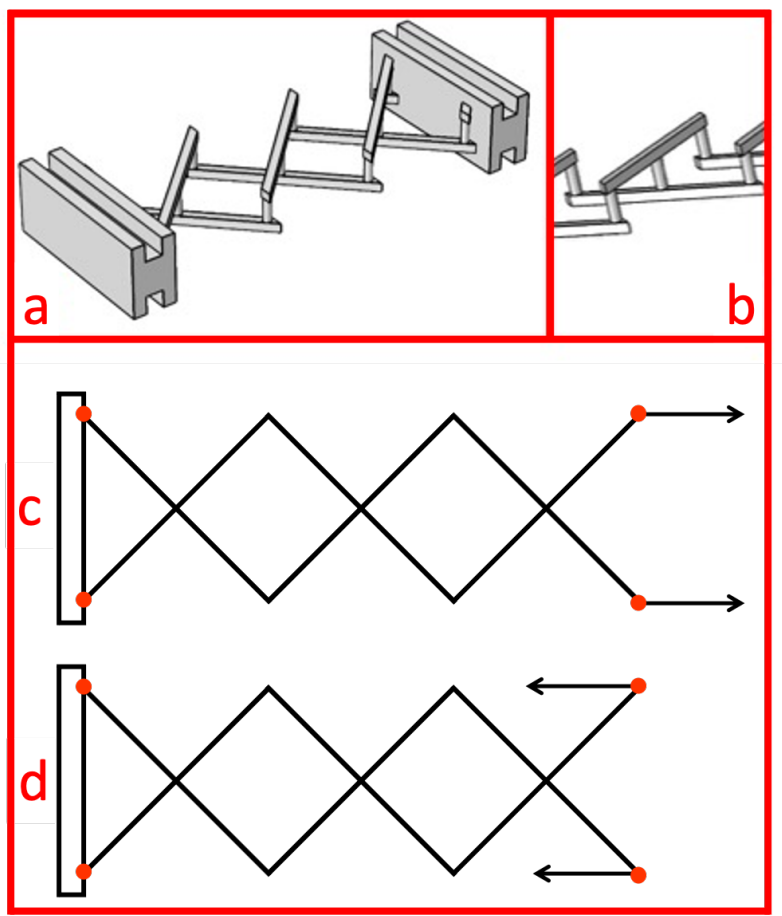

Figure 1: Minimal pantographic structure consisting of three successive cells. CAD model used for $3 \mathrm{D}$ printing the specimen (a) and detail of the pivots (b). The test studied in this work are a bias extension test (c) and a compression test (d).

In addition to macroscopic second gradient models of pantographic structures, mesoscopic descriptions are based on nonlinear Euler-Bernoulli beams for describing the "arms" of the pantograph. This mesomodel has been successfully used for interpreting experimental measurements (e.g. bias extension tests) performed on a fabriclike pantograph 2. In the following, the mesomodel was selected in the analysis of pantographic cells. The very low number of unit cells does not seem sufficient for justifying the use of a macroscopic (i.e. continuum) model [19. In order to validate second gradient models used to describe such metamaterials, it is necessary to conduct experimental tests. It is therefore essential to produce samples to be used in experiments. This has been significantly simplified by the rapid development of Additive Manufacturing.

From a modeling point of view, one can make appropriate simplifying choices depending on the type of mechanical behavior one expects for a given test. If, for example, one expects that the chosen test does not involve out-ofplane deformations, then one can model the pantographic structure with a 2D model, neglecting deformation mechanisms characteristic of out-of-plane deformations (such as torsion of the fibers or their bending in the direction orthogonal to the plane in which they are initially contained). In a 2D model, the deformations of the fibers are taken into account by introducing in the deformation energy a term related to elongation and one related to bending. Pivots, on the other hand, are a more complex structural element: in order to observe experimen- 
tally the energy contributions related to the second gradi-174 ent of the displacement (i.e. those related to the bending ${ }_{175}$ of the fibers) it is necessary that the deformation energy of the pivots is as small as possible. So the ideal would be to have perfect hinges. This is of course possible in numerical simulations, but it is not always feasible in the production of test specimens (the possibility of producing perfect working hinges depends very much on the scale at which such mechanisms are printed and on the precision of the 3D printer). So in some cases, as in the present one, it is necessary to take into account the torsion of the cylinders, as well as their shear deformation, which becomes non-negligible when they are slender. If, on the other hand, the ratio between the height and diameter of the pivot is very small, the torsion energy prevails over the bending energy and makes the second gradient contribution negligible. From a microstructure homogenization ${ }_{176}$ point of view, the continuum obtained in this case is a first ${ }_{177}$ gradient continuum as shown in 5 .

In this paper, the mechanical behavior of pantographic ${ }_{179}$ cells is analyzed. The work is organized as follows: (i) the ${ }_{180}$ adopted model, which was introduced in Ref. 2, is recalled ${ }_{181}$ and adapted to pantographic cells; (ii) through the model, ${ }_{182}$ numerical simulations are performed and the consequent ${ }_{183}$ results (force-displacement curves and deformed shapes of ${ }_{184}$ the structure) are compared to experimental data relative ${ }_{185}$ to bias extension and compression tests; (iii) by means of ${ }_{186}$ a newly conceived Digital Image Correlation (DIC) frame- ${ }_{187}$ work [19], a theoretical "Ansatz" about the nature of de- 188 formations in the hinges is successfully corroborated. $\quad{ }_{189}$

Finally, we want to remark that in this work we have expressly chosen to study a pantographic structure consisting of only three cells. In fact, with larger structures, it would be difficult to study the various energy terms precisely and to separate the pivot terms. Clearly, the results obtained for a structure consisting of a few cells can also be used when one wishes to study a structure consisting of many cells. Note, however, that when the number of cells becomes very high, the continuous model can be used for pantographic structures. The "mesoscopic" model presented in this paper is optimal in the case of structures with a wide knit.

\section{Euler-Bernoulli beam theory adapted to panto- graphic cells}

In this section, the model presented in Ref. [2] is discussed and adapted to the structure under study. First, a summary is given of the physical system to be designed, and then a description is provided of how to proceed in modeling the observable quantities of the system. The interested reader will find additional details of the beam system presented herein in Refs. 22 .

As stated in the introduction, the real system that is ${ }_{100}$ the object of this work is a pantographic structure, which consists of an assembly of two planar alignments of parallel ${ }_{192}$ fibers (or beams), see Figure 2 It was produced by Additive Manufacturing and was made of polyamide PA2200.

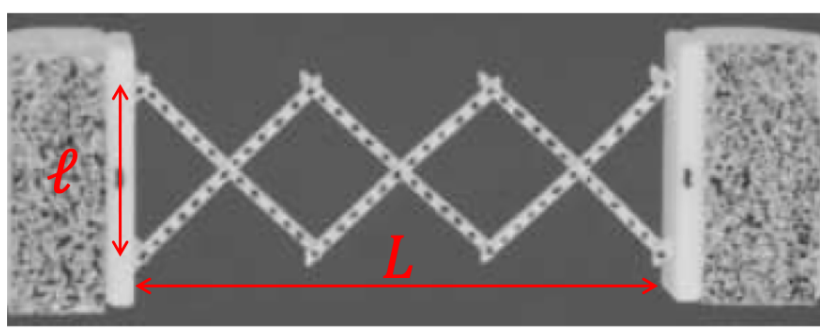

Figure 2: Picture of the reference configuration of a printed pantographic modular structure $(\ell=10 \mathrm{~mm}$ and $L=30 \mathrm{~mm})$.

The geometrical and material characteristics of the sample are gathered in Tables 1. 2. In this specific case, the pantographic structure is referred as 'modular' because its overall dimensions (i.e. $\ell$ and $L$, see Figure 2 ) are of the order of centimeters. In this particular case, the overall dimensions are $10 \mathrm{~mm} \times 30 \mathrm{~mm}$. It consists of three basic pantograph cells with an X-shape. This structure represents the prototype of a minimal pantographic structure because, in order to observe its typical phenomenology, it is necessary to consider at least three $\mathrm{X}$ cells, two of which (i.e. the exterior ones) being clamped at their ends. The beams that constitute the pantograph are connected at their points of intersection by means of deformable cylinders that behave like hinges.

Table 1: Mechanical properties of the 3D printed polyamide PA2200

\begin{tabular}{l}
\hline Mechanical Properties \\
\hline Young modulus $E=0.5 \mathrm{GPa}$ \\
Poisson ratio $\nu=0.33$ \\
Shear modulus $G=\frac{E}{2(1+\nu)}$ \\
\hline
\end{tabular}

Table 2: Geometric dimensions of the beams composing the pantographic cells.

\begin{tabular}{l}
\hline Geometrical properties \\
\hline Basis of the cross section $b=1 \mathrm{~mm}$ \\
Height of the cross section $h=1 \mathrm{~mm}$ \\
Cross sectional area $A=b \times h$ \\
Moment of inertia $J=\frac{1}{12} b h^{3}$ \\
Height of the pivots $H_{P}=2 \mathrm{~mm}$ \\
Radius of the pivots $R_{P}=0.45 \mathrm{~mm}$ \\
\hline
\end{tabular}

The deformation observed experimentally can be schematized as follows, namely, the deformation of the beams, which are stretched and bent, and the deformation 
of the hinges. The latter ones are subject to torsion due 235 to the relative rotation of the beams of the two families, 236 and to shear deformation. The effect of torsion is observed 237 by comparing the reaction force plot measured versus pre-23в scribed displacement when the hinges are elastic with a239 certain torsional stiffness and when they are perfect ( see $_{240}$ for example Ref. 27]). In this last case, the initial part of 241 the plot is close to zero for the forces. When the hinges 242 deform, the force departs from 0 . The direct observation 243 of the shear deformation mechanism of the hinges was de-244 duced through Digital Image Correlation [19] as also shown 245 below.

\subsection{Mechanical model}

The model adopted herein to describe the fiber system ${ }^{249}$ discussed above has been introduced in Ref. 2 and is sum-250 marized as shown in Figure 3 It characterizes the fibers as ${ }^{251}$ Euler-Bernoulli (nonlinear) beams, whilst the hinges are ${ }^{252}$ modeled as rotational springs (torsion model of the real ${ }^{253}$ cylinders) in addition to extensional springs (to take into ${ }^{254}$ account shear, which produces relative displacements be-255 tween the two fibers connected by the hinge itself). It was ${ }^{250}$ observed in previous works [2, 26] that for ratios between ${ }^{257}$ the height and radius of the hinge in a certain range, the ${ }^{258}$ hinge may exhibit, in addition to the usual torsional de-259 formation due to the change of angle between the fibers of ${ }^{260}$ the two families, deformations due to the relative motion ${ }^{261}$ of the two fibers, which was modeled by a linear spring..262 It is assumed that the constitutive law of the extensional ${ }^{263}$ spring, modeling the hinge in terms of energy, depends ${ }^{264}$ the square of the relative displacement. Another cubic de-265 pendence on the relative displacement must also be added ${ }^{260}$ to obtain results in good agreement with experimental ob-267 servations. This last deformation mechanism allows relative sliding to be introduced between the fibers of the two families in correspondence of the hinges. For this reason, the associated energy term is called fiber connectivity.
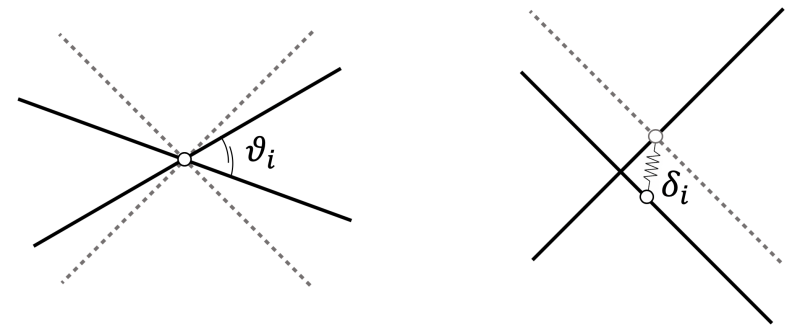

In Ref. 2], it was shown how, in order to obtain the de-280 formed configuration and the reaction forces on the edges ${ }_{281}$ of the pantographic structure, the strain energy of the ${ }_{282}$ structure had to be written in terms of its individual con-283 stituents. Specifically, if each beam is considered as com-284 posed of a number of finite elements corresponding to the ${ }_{285}$
Figure 3. Kinematics of hinges depicting torsion (left) and fiber con- ${ }^{276}$ nectivity (right) mechanisms. number of beam elements between two consecutive hinges (in the simple case of pantographic cells, all beams are made up of two finite elements), then the strain energy of each element is given by extensional and bending contributions. In order to obtain the strain energy of the entire structure, all these energies of the finite elements are combined. It is also necessary to add the contribution of the hinges. For each hinge, there is a term due to torsion (which in the case of perfect hinges should vanish), and another one due to possible shear. These contributions to the strain energy are as numerous as the number of hinges in the pantographic structure. For a pantographic modular structure, the number of beam elements and the number of hinges is easily counted. For each fiber family, there are three fibers, each consisting of two elements, for a total number of 12 fiber elements for 7 hinges.

Let us call the ends of each beam element nodes. At these specific points, which correspond to the hinges, the nodal displacements and rotations are defined for the transverse sections 2, and are used to write the strain energy of the beams/fibers and the hinges. These displacements and rotations are therefore the unknowns to the problem, and are obtained by prescribing boundary conditions and minimizing the total strain energy. In the present case, which aims to analyze bias extension and compression tests, the boundary conditions consist in zero displacements for the nodes on one of the two short sides of the pantographic structure (corresponding to the clamped side), and in assigning displacements to the nodes on the other short side. The horizontal component of the displacements is designated as $U_{a}$ and $U_{b}$ ( $a$ and $b$ for the two fiber families), and the vertical ones $V_{a}$ and $V_{b}$, then the boundary conditions become

$$
\begin{array}{r}
U_{i}(0, j)=0, U_{i}(L, j)=\bar{U}, \\
\forall i \in\{a, b\}, \forall j \in\{\text { set of nodes in the short side }\} \\
V_{i}(0, j)=0, V_{i}(L, j)=0, \\
\forall i \in\{a, b\}, \forall j \in\{\text { set of nodes in the short side }\}
\end{array}
$$
of the pantographic architecture is non-linear, as is specified in the following. Furthermore, the geometric arrangement of the fibers itself provides for a global energy that is non-linear. The terms that contribute to the strain energy of the fibers are: (i) elongation energy, written in terms 


\subsection{Ritz approach for minimizing the energy}

The strain energy is written as the sum of the contributions of beam elements for extensional and flexural terms, as well as hinges for torsional and fiber connectivity terms. Specifically, let $N$ be the number of beam elements, and $P$ the number of hinges (for the studied pantographic structure, $N=6$ for two families of beams, and $P=7$ ), the extensional energy reads

$$
\mathcal{E}^{(e)}=\sum_{i=1}^{2 N} \frac{1}{2} \int_{0}^{\lambda_{i}} E A \eta^{2}(x) d x
$$

and the flexural energy

$$
\mathcal{E}^{(b)}=\sum_{i=1}^{2 N} \frac{1}{2} \int_{0}^{\lambda_{i}} E J \chi^{2}(x) d x
$$

where $\eta(x)$ and $\chi(x)$ denote generalized strains relative to the axis line of the beam. More precisely, $\eta(x)$ is the stretching strain, while $\chi(x)$ is the curvature. For the $\mathrm{e}_{313}$ hinges, the torsional term depends on the angular vari- ${ }_{314}$ ation between the two fibers that the hinge interconnects ${ }_{315}$

$$
\mathcal{E}^{(p)}=\sum_{i=1}^{P} \frac{1}{2} \mathbb{K}_{p}\left(\frac{\pi}{2}-\Delta \vartheta_{i}\right)^{\beta}
$$

where $\mathbb{K}_{p}$ accounts for the torsional stiffness and is related ${ }_{320}$ to the geometric characteristics of the hinge, $\Delta \vartheta_{i}$ the variation of the angle between the fibers crossing in the $i$-th hinge, $\beta$ an exponent that in general is different from $2^{321}$ and is obtained from fit of experimental data. The energy ${ }^{322}$ term related to fiber connectivity is written as

$$
\mathcal{E}^{(c)}=\sum_{i=1}^{P} \frac{1}{2} \mathbb{K}_{c}\left(\delta_{i}^{2}+\mathfrak{K}_{3} \delta_{i}^{3}+\ldots\right)
$$

in the undeformed configuration are superimposed (i.e. $\left.\left(X_{A}, Y_{A}\right)=\left(X_{B}, Y_{B}\right)\right)$. A deformation of the structure may result in a relative displacement of the hinge ends. By assigning the horizontal and vertical nodal shifts for the two families $\left(U_{A}, V_{A}\right)$ and $\left(U_{B}, V_{B}\right)$, the relative shift of the fibers $\delta_{i}$ is expressed in terms of these positions and shifts as

$$
\begin{aligned}
\delta_{i}^{2} & =\left[\left(X_{A}^{(i)}+U_{A}^{(i)}\right)-\left(X_{B}^{(i)}+U_{B}^{(i)}\right)\right]^{2} \\
& +\left[\left(Y_{A}^{(i)}+V_{A}^{(i)}\right)-\left(Y_{B}^{(i)}+V_{B}^{(i)}\right)\right]^{2}
\end{aligned}
$$

Like $\mathcal{E}^{(c)}$, the other energy terms are also written according to the components of displacements and rotations of the nodes. It suffices to minimize the total strain energy to find, for each assigned deformation step, the nodal displacements and rotations. With these variables and the shape functions used to define finite elements, the deformed configuration is obtained, and then the reaction forces. The selected shape functions are cubic Hermite polynomials. The detailed procedure of finite element decomposition and minimization is reported in Ref. 2. Let us merely indicate the way to obtain the values of the displacements at equilibrium. The total strain energy reads $\mathcal{E}$

$$
\mathcal{E}=\mathcal{E}^{(e)}+\mathcal{E}^{(b)}+\mathcal{E}^{(p)}+\mathcal{E}^{(c)}
$$

and it is postulated that equilibrium is given by the minimum of the potential energy

$$
\delta \mathcal{E}=0
$$

In the present case, there is no need for adding an external work to the minimization because essential boundary conditions were prescribed.

In the next section, the results of numerical simulations based on the presented model are reported, and comparisons with experimental measurements are carried out. All details for the numerical implementation of the presented model is found in Ref. [2].

\section{Numerical simulations and comparison with ex- perimental measurements}

The experimental tests have been performed with quasi-static loading conditions: the loading speed is $0.5 \mathrm{~mm} / \mathrm{s}$. Moreover, we want to underline that the fiber element and hinge numbers, in general, can affect the accuracy and convergency of the numerical results. The number of hinges considered in the code used corresponds to the number of physical pivots that are present in the tested specimen. On the other hand, the numerical code we wrote to perform the simulations subdivides the individual fibers into non-linear Euler-Bernoulli beam finite elements. As we have already remarked, the nodes of the subdivision into finite elements correspond to the physical pivots. One could certainly choose to increase the number of nodes and finite elements. This increases the computational effort 
considerably. The calculation time in the current case is only a few seconds. By increasing the number of nodes, the calculation time can also increase considerably. The result, however, does not change substantially, and, therefore, that the chosen decomposition is sufficient for the present case.

Figures 4 and 5 show the comparison between the measured reaction forces and those calculated for the extension and compression tests at the end of the calibration of the four parameters of the above model.

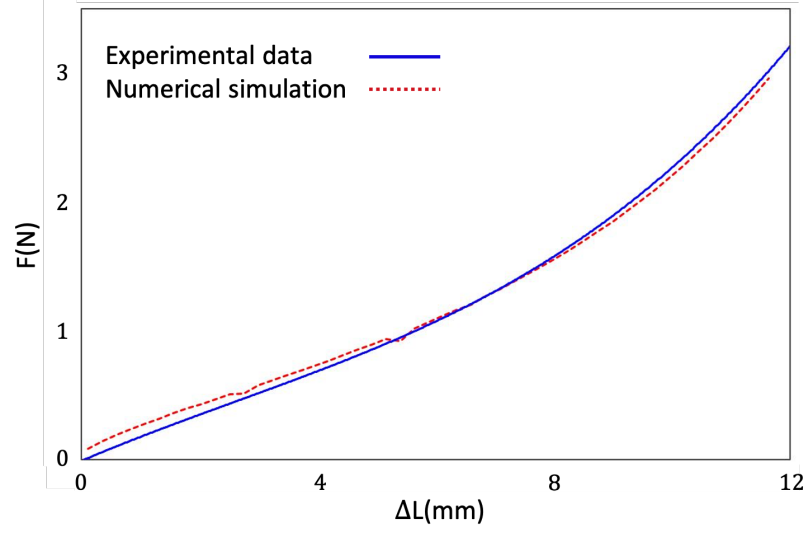

Figure 4: Bias extension test. Comparison between experiment (blue ${ }^{380}$ solid line) and model prediction (red dotted line).

These macroscopic predictions are in good agreement 383 with the experimental results. To obtain them, some of 384 the constitutive parameters have been calibrated. More 385 specifically, the elongation and bending stiffness were as-386 sumed to be known from the Euler-Bernoulli beam theory.387 They are expressed in terms of the Young's modulus of 388 the chosen material and of geometrical dimensions of theзвя beams.

As we have specified, the parameters of the model were 31 calibrated on the extension test, whose force-displacement 392 graph is shown in Figure 4. The same parameters, thus 393 calibrated expressly for the extension test, were used to394 carry out the numerical simulation of the compression test. 395 Now, of course, in the case of Figure 5 the agreement be-396 tween numerical simulation and experimental data is not397 as strong as in Figure 4, but the results are reassuring. ${ }^{398}$ Clearly better results could be obtained by an iterativeз99 calibration that takes into account the experimental data 400 of both tests: as far as the present study is concerned,401 we simply observe that the model adopted also predicts $\mathrm{a}^{402}$ lesser involvement of the fiber-connectivity mechanism in ${ }^{403}$ the compression test, a fact that is also revealed by the ${ }^{404}$ analysis of the experimental images carried out by means 405 of Digital Image Correlation techniques. This last circum-406 stance will be clearer in the following.

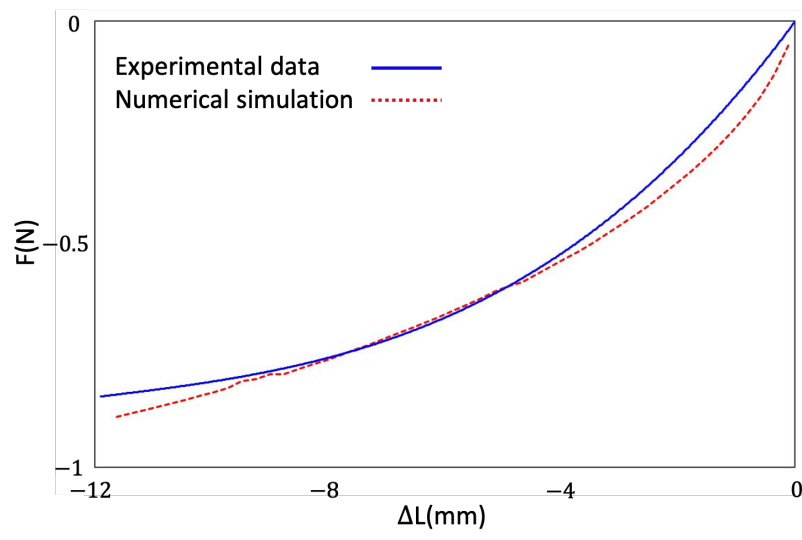

Figure 5: Bias compression test. Comparison between experiment (blue solid line) and model prediction (red dotted line).

As it can be seen in the following Fig. 6, the elongation and bending energies of the fibers are much smaller than the energies associated with the pivots. The largest energy contribution is due to the torsion of the pivots and, in a second step, to the fibre connectivity energy term. The latter two terms are clearly non-linear, although, as we mentioned earlier, all four energy contributions are nonlinear and therefore partly contribute to the non-linearity found in the curves in Figures 4 and 5 . Furthermore, we would like to point out that the non-linear character of the force-displacement graphs is not due to the material of which the specimen is made, but to the architecture according to which the fibers are organized. In the considered tests, no plasticization phenomena are detected: the fibers definitely did not undergo plasticization because they are not sufficiently elongated (or compressed) and not sufficiently flexed; the pivots could plasticize, but in that case plasticization would be easily observable in the force-displacement graph (for example this is well visible in metal specimens, as shown in [27]). Furthermore, the specimen was unloaded and no permanent deformation was visible.

The parameters related to the hinges are those that need to be calibrated since the two energy components related to the hinges were deduced phenomenologically. The calibration was performed by comparing the computed force-displacement curve with the experimental one for the bias extension test (see Figure 4). As the study we present in this paper consists of a qualitative analysis of the mechanical behavior of a minimal pantographic structure in order to investigate the individual mechanisms occurring at the level of the microstructure, the model parameters are optimized "by hand" in order to obtain both a force-displacement curve compatible with the experimental one and deformed shapes as close as possible to those observed experimentally. As we have already pointed out, in the second part of the paper, some analyses carried out by means of Digital Image Correlation are presented. The perspective of the joint work of the various co-authors is to obtain a numerical tool able to quantitatively calibrate the 
parameters of the model on the basis of the experimental data. In this perspective, the use of Digital Image Correlation is central. Above all, the authors' intent is to obtain an Integrated Digital Image Correlation 20, that can interact in an automated way with the numerical program and obtain the most suitable values for the constituent parameters of the model. At present, this is not yet implemented and requires a very articulated work, which the authors are carrying out. The calibrated parameters are reported in Table 3. An important point to note is that the same constitutive parameters were used in the model to describe tension and compression tests.

Table 3: Constitutive parameters of the studied pantographic cells.

\begin{tabular}{lr}
\hline Parameter & Value \\
\hline Shear stiffness $\mathbb{K}_{p}$ & $4.60 \cdot 10^{-3} \mathrm{~N} / \mathrm{m}$ \\
Fiber connectivity stiffness $\mathbb{K}_{c}$ & $1.20 \cdot 10^{3} \mathrm{~N} / \mathrm{m}$ \\
Fiber connectivity correction $\mathfrak{K}_{3}$ & $1.00 \cdot 10^{2} \mathrm{~N} / \mathrm{m}^{2}$ \\
Exponent in shear energy $\beta$ & 1.55 \\
\hline
\end{tabular}

With the calibrated parameters, the contributions of the various parts of the strain energy were computed for the two considered experiments (Figure 6). In these plots, in addition to the total energy (black solid line), the different contributions are shown, namely, stretching energy ${ }^{451}$ (red dashed line), bending energy (green dotted line), ${ }^{452}$ shear energy (blue dashed line) and fiber connectivity en- ${ }^{453}$ ergy (yellow dot-dashed line). It is observed that the total ${ }^{454}$ energy in extension is about twice as high as that in com- $^{455}$ pression for the same elongation magnitude. In both tests, ${ }^{456}$ the prevalent component is shear (i.e., most of the strain ${ }^{457}$ energy is due to hinge torsion).

The difference between the two total energies lies in the ${ }^{459}$ fact that the deformation mechanisms mainly involved in ${ }^{460}$ extension and compression are not identical. In an exten- ${ }^{461}$ sion test, there is a non-negligible component of the fiber ${ }^{462}$ connectivity energy, while in a compression test this com- ${ }^{463}$ ponent is of the same order of magnitude as the bending ${ }^{464}$ energy. In both tests, as expected from a theoretical point ${ }^{465}$ of view, the stretching energy is negligible. Further, the ${ }^{466}$ levels of shear energy are very similar for both tests when ${ }^{467}$ compared to other contributions. This observation reveals that in both tests the relative rotation of the fibers in correspondence of the hinges is roughly the same, thereby revealing a certain "symmetry" between the two tests. The lack of overall symmetry at the global scale is then interpreted as the result of fiber connectivity.

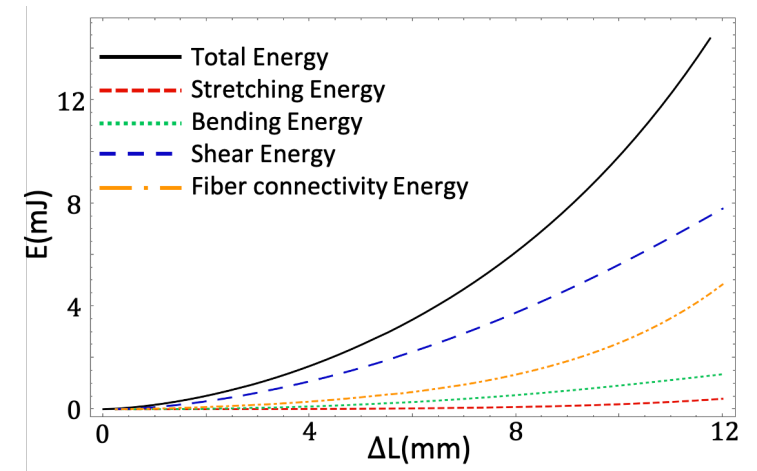

(a)

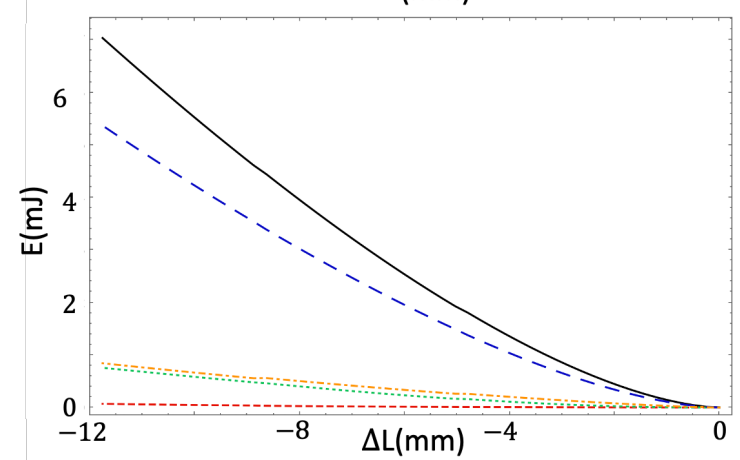

(b)

Figure 6: Energy contributions for bias extension (a) and compression (b) tests.

In Figures 7. 9, the deformed configurations of the pantographic cells are compared to the simulated shapes for different deformation steps. More precisely, in Figure 7. a qualitative comparison between experiment and simulation was carried out for the bias extension test. The deformed shapes were numerically determined using Hermite cubic polynomials, which were mentioned in the previous section. The order of such shape functions was sufficient to ensure that the global deformed shape and that the calculation of the reaction force were well predicted (Figure 4). For the computation of the internal actions in beams (i.e. local analysis of deformation), the chosen order of the shape functions was not sufficient to obtain reliable results. In order to numerically calculate the internal actions, it would be desirable to choose shape functions of higher order or, alternatively, to increase the number of finite elements to discretize each beam. 


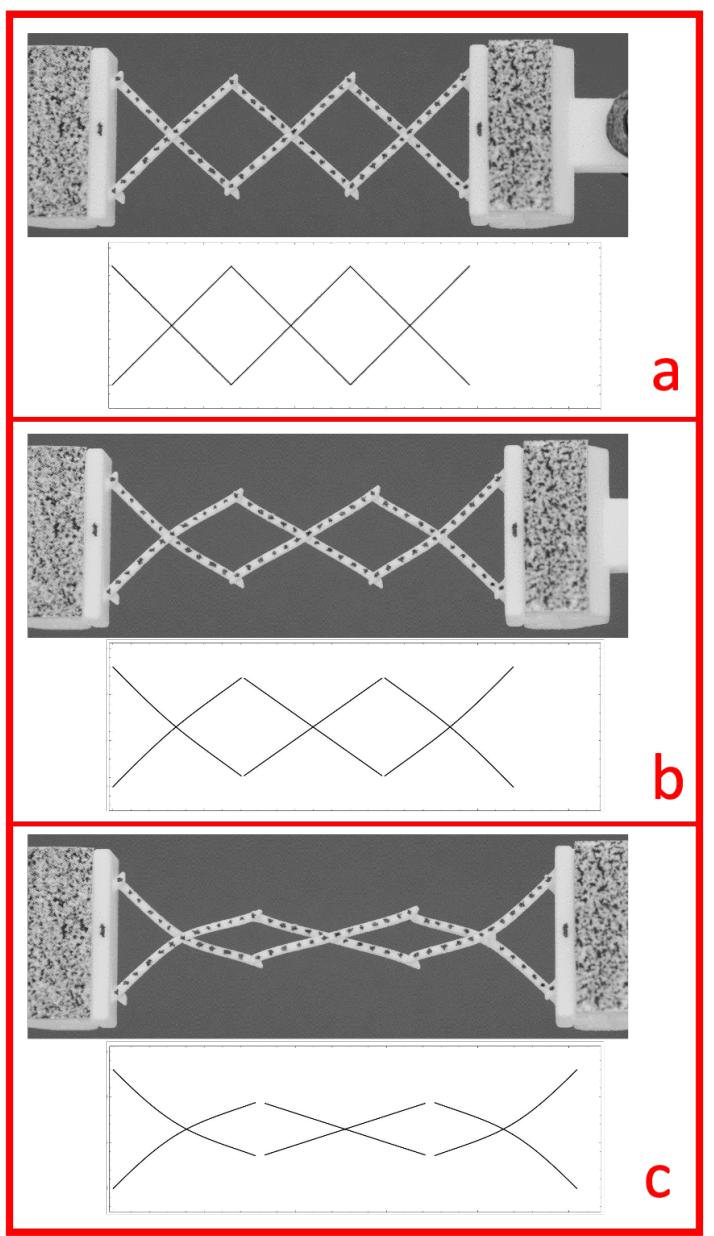

Figure 7: Bias extension test: comparison between experimental deformed shapes and the numerically simulated ones for three selected values of prescribed displacement. Imposed displacement on the horizontal axis and measured reaction force on the vertical axis.

In Figure 8 , the same type of comparison is performed for the compression test. As it was clear by observing Fig- ${ }_{491}$ ure 6, extension and compression tests were not completely $y_{492}$ symmetric, due to the fiber-connectivity energy term. If,$_{493}$ on the one hand, this is simply accountable from the model ${ }_{494}$ point of view (in fact the fiber-connectivity energy term $\mathrm{m}_{495}$ depends also on the cube of the relative displacement), $\mathrm{on}_{496}$ the other hand one has to carefully interpret this lack of ${ }_{497}$ symmetry in the context of the experiments. Figure 7. 8 only show the face view of the two experiments. It is likely that, in the bias extension test, the deformation of the specimen is always in the plane. On the contrary, the specimen deformations are unlikely to remain in-plane in the compression test. For example, in Figure 8(c), the relative displacements of the four hinges at the ends of the central cell seem very pronounced, but they could be affected also from parallax induced by out-of-place deformations (specifically, by torsion of the fibers themselves). This point will be further discussed when dealing with the comparisons between computed and measured relative displacements.

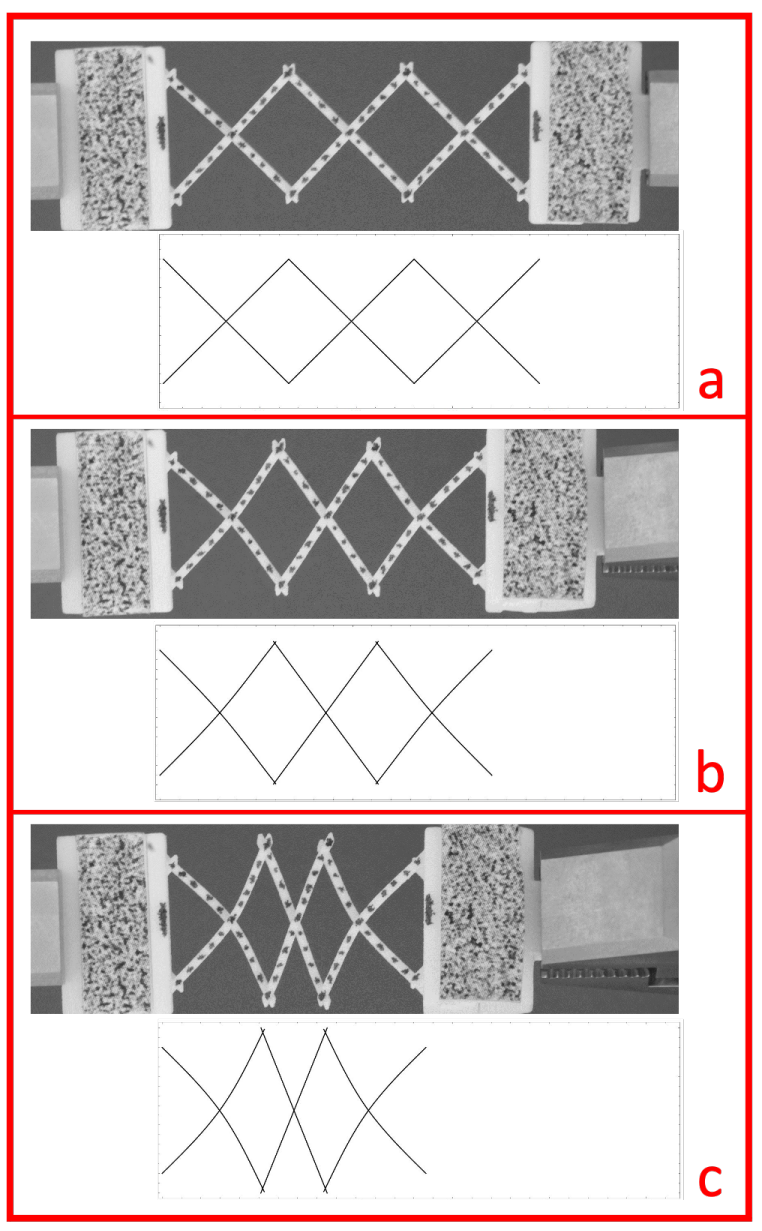

Figure 8: Bias compression test: comparison between experimental deformed shapes and the deformed shapes obatined by numerical simulation for three selected values of prescribed displacement.

In Figure 9, the predicted deformed shape (in red) is laid over the image of the last deformation step for both tests. This result probes in a more quantitative way the trustworthiness of the computed deformed shapes, which are in very good agreement with experimental observations. An even more quantitative comparison would be possible using Digital Image Correlation (see Section 4 ). This kind of comparison has been recently performed in Ref. 4 . 


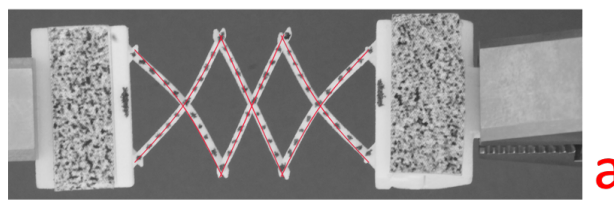

a

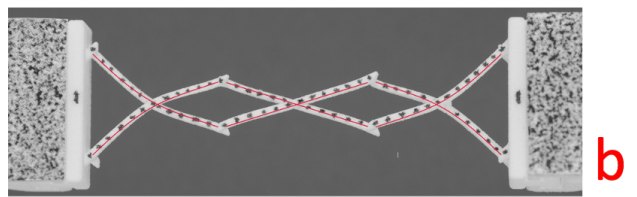

Figure 9: Superposition of computed (red) and experimental deformed shapes at the end of bias compression (a) and extension (b) tests.

In Figures 10 12, additional details about the numeri-

cal results related to the fiber connectivity are shown. This contribution was introduced in the strain energy in order to take into account the slender ratio between height and radius of the hinges. The relative displacements of the beams in correspondence of the hinges, which are referred to as displacement jumps, are indicated by the distance of the two red and blue points that correspond to the upper and lower ends of the hinges. A symmetry is observed in these displacement jumps. In Figure 10 , the fiber connectivity is studied for four different deformation steps for the bias extension test, analogous results are reported for the compression test in Figure 11 .

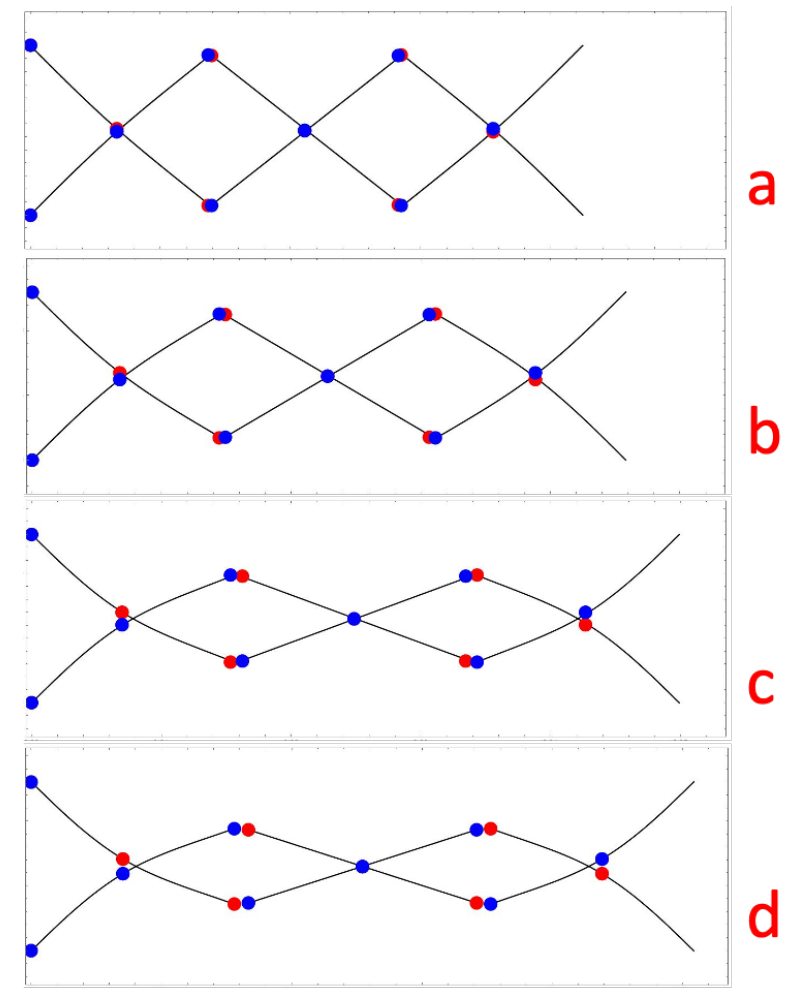

Figure 10: Bias extension test: numerically computed deformed shapes of the structure. The red and blue points, which are superimposed in the undeformed configuration, represent the two ends of the hinges and their subsequent misalignment is the result of the fiber connectivity term.
Two aspects emerge from the observation of Figures 10 and 11. In both tests, for reasons of symmetry, the central hinge is not sheared at all (and for the same reasons, it is also the hinge that experiences the maximum relative rotation between the beams). The displacement jumps calculated in the bias extension test are in amplitude greater than those of the compression test, even if the absolute range of deformation is the same for both tests. The second observation is a direct consequence of the fact that the energy term associated with the fiber connectivity is not quadratic but consists of quadratic and cubic terms. In the next section, this asymmetry is experimentally probed. 


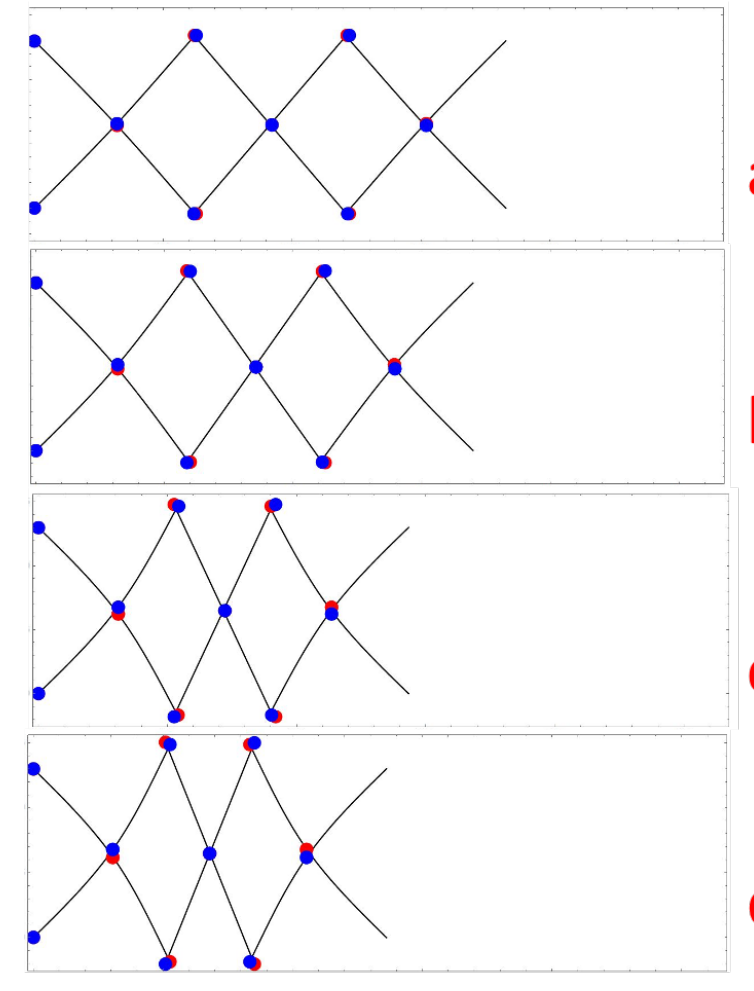

b

C

Figure 11: Bias compression test: computed deformed shapes of the cells. The red and blue points, which are superimposed in the undeformed configuration, represent the two ends of the hinges and ${ }^{543}$ their subsequent misalignment is the result of the fiber connectivity 544 term.

In Figure 12, for the last deformation step of the bias extension test, the displacement jumps are highlighted by adding yellow arrows. These arrows have the advantage of indicating not only the amplitude of the displacement jump, but also its direction. A careful observation of these arrows shows that some displacements, which would otherwise appear completely horizontal, have a vertical component as well. An analysis of these displacement jumps in a more quantitative way is reported in Figures 14.15.

For the hinges labelled in Figure 13 , displacement jumps are plotted in components for the bias extension test in Figure 14 and for the compression test in Figure 15.

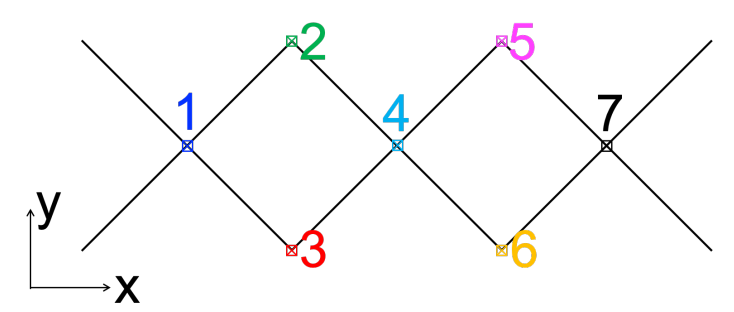

Figure 13: Location of the 7 hinges where the relative displacement has been computed for a comparison with the measured values obtained via DIC.

In Figures 14 15 the displacement jumps at the hinges as labelled in Figure 13 are plotted for the two numerical simulations (respectively, bias extension and compression). These plots show that there is a certain symmetry in the microshear deformation of the hinges. Observing Figures 14, 15, in both tests the hinges behave similarly. Specifically, the hinges labelled 2, 3, 5 and 6 mainly experience horizontal displacement jumps, while hinges 1 and 7 are sheared along the $y$ (vertical) direction. Last, for symmetry reasons, the central hinge, labelled 4 does not undergo any displacement jump.

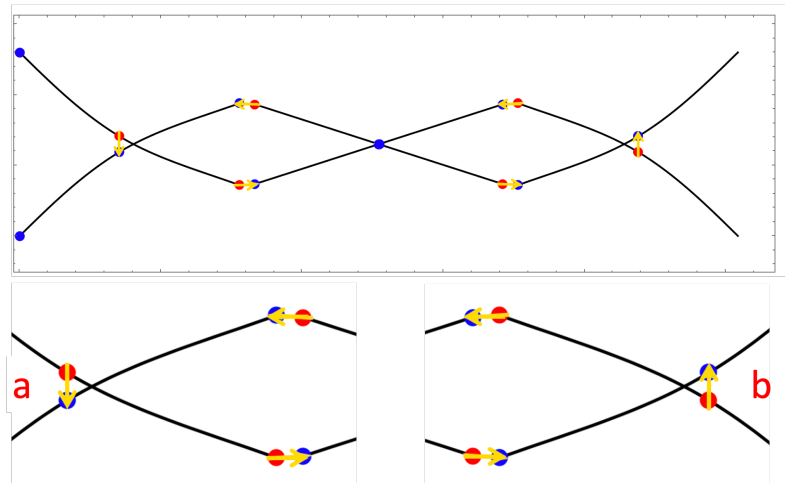

Figure 12: Numerically computed deformed shape of the pantographic cells for the last extension step. The direction of the relative displacement between the beams of the two families is depicted by yellow arrow. In (a) and (b) the extremes of the figure are magnified for showing the antisymmetry of the arrows.
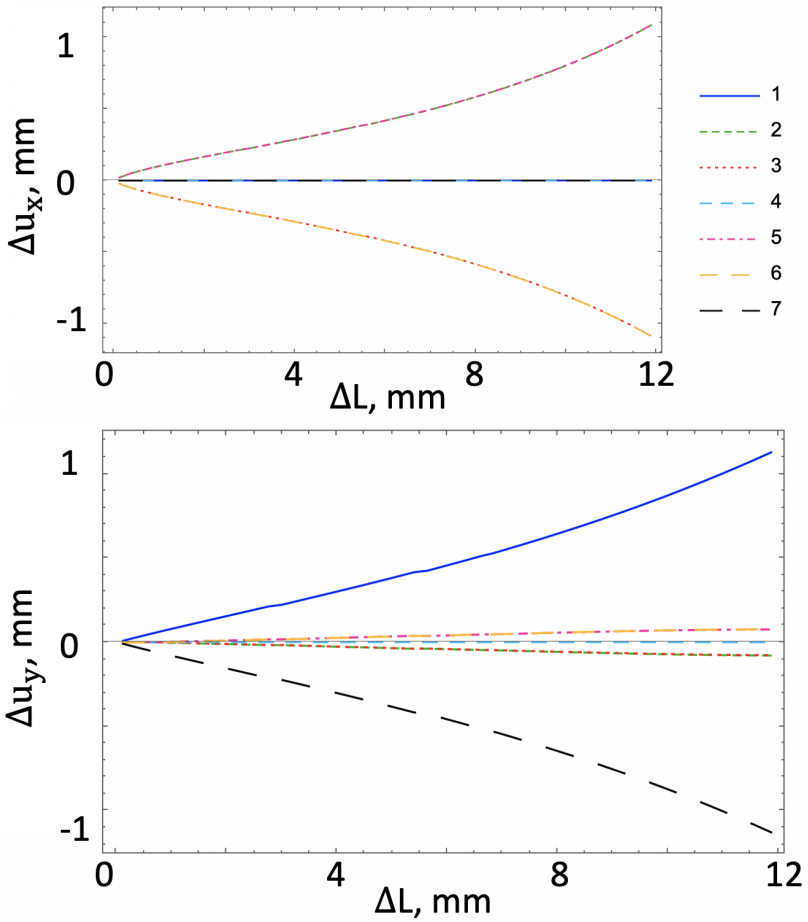

Figure 14: Predicted history of displacement jump for the 7 labelled pivots in the horizontal (up) and vertical (down) directions for the bias extension. 

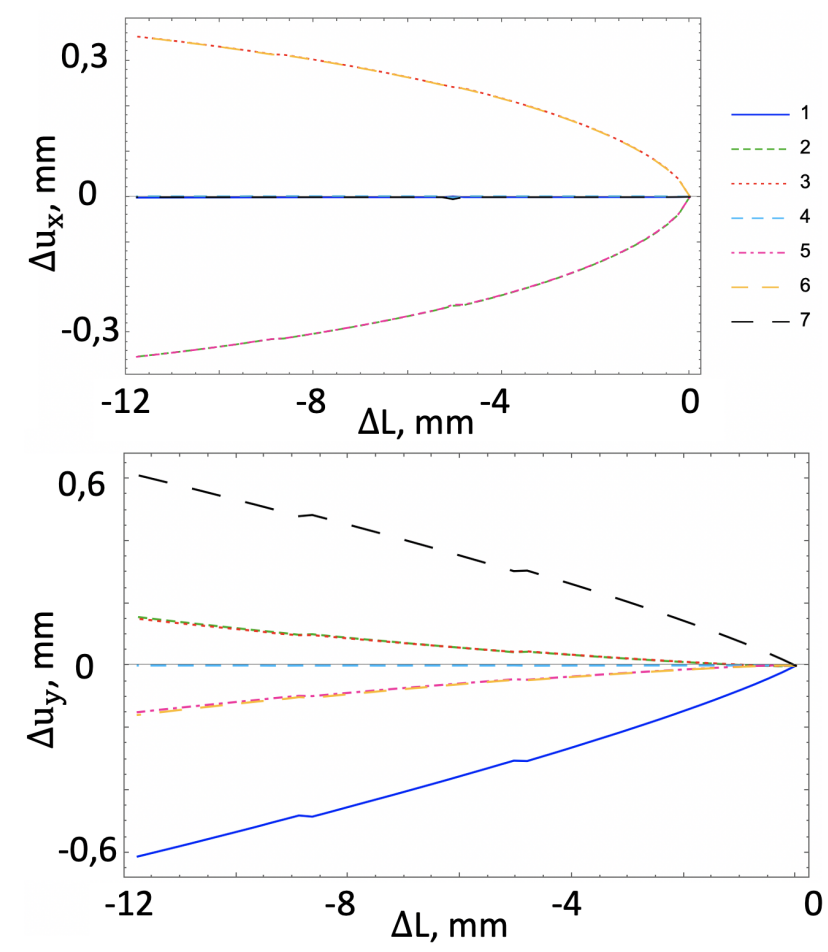

Figure 15: History of displacement jump for the 7 labelled pivots in the horizontal (up) and vertical (down) directions for the compres- ${ }^{598}$ sion test numerical simulation.
A further study could investigate the distribution of 602 the displacement jumps for pantographic structures with 603 more cells. A deeper analysis should be dedicated to the 604 study of these jumps in multi-layered pantographic struc-605 tures (which until now have been considered only for their ${ }_{600}$ performing properties in three-point bending tests [29]).

Before presenting the results obtained by DIC, we want to remark that the discrepancies that will be observed between the Figs. 14 15 and the Figs. 16 17 can be attributed to many simplifications assumed in the present study, as, for example, the fact that both in the numerical model and in the DIC analysis are not taken into account out-of-plane deformations, which can clearly influence the shear of the hinges. Out-of-plane deformations are non prevalent in the presented experimental tests. This, of course, does not avoid that such deformations can occur and, indeed, can be observed in some tests, expressly designed for the study of such a phenomenon. However, we have to consider some aspects: out-of-plain deformation and buckling are phenomena that can be studied by means of stability criteria of a structure. In the case of the tests presented here, an out-of-plain deformation may occur during the compression test. The main deformation mechanisms involved in the compression test are those related to fibre bending and pivot torsion and shear. In particular, it has been observed by some co-authors of this work (in a paper that is currently being written) that the torsional stiffness of the pivots greatly influences the presence or absence of out-of-plane deformation.

\section{Digital Image Correlation analyses}

In the previous section, the acquired pictures were used for qualitative comparisons between the deformed shapes observed experimentally and predicted numerically with the calibrated model (Figures 7 9). These images can also be used to measure displacement fields via Digital Image Correlation 28]. Of the various approaches applied to the bias extension test [19, microscale analyses were run in which each beam was meshed and backtracked to fit the reference configuration of each test. Since displacement jumps were sought, no constraints (e.g. via Lagrange multipliers) were applied to the displacement of the connecting ends of each hinge. These kinematic hypotheses led to the lowest registration residuals and thus were deemed the closer to the experiment [19.

Figure 16 shows the displacement jumps for the bias extension test. For the longitudinal component (Figure 16(a)), the overall trends are identical to those obtained by numerical simulation (Figure 14), namely, the two lower hinges (3 and 6 ) experience positive jumps, the two upper hinges (2 and 5) undergo negative displacement jumps, the two extreme hinges (1 and 7 ) have virtually no displacement jumps. Conversely, the middle hinge experiences some negative displacement jump in both directions, which was not expected from the numerical simulation. This observation shows that the response of the pantographic cells did not possess all the symmetries found in the simulation. This is due to imperfections induced by the printing process of such small structures, and alignment of the sample in the testing machine. This trend is further confirmed when comparing the vertical component of the displacement jumps (Figures 14 and 16 (b)). 


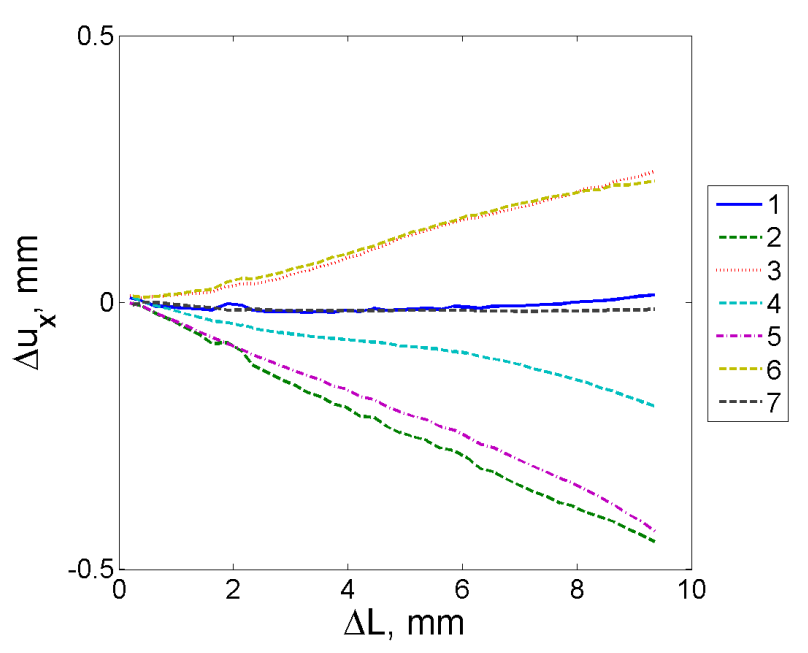

(a)

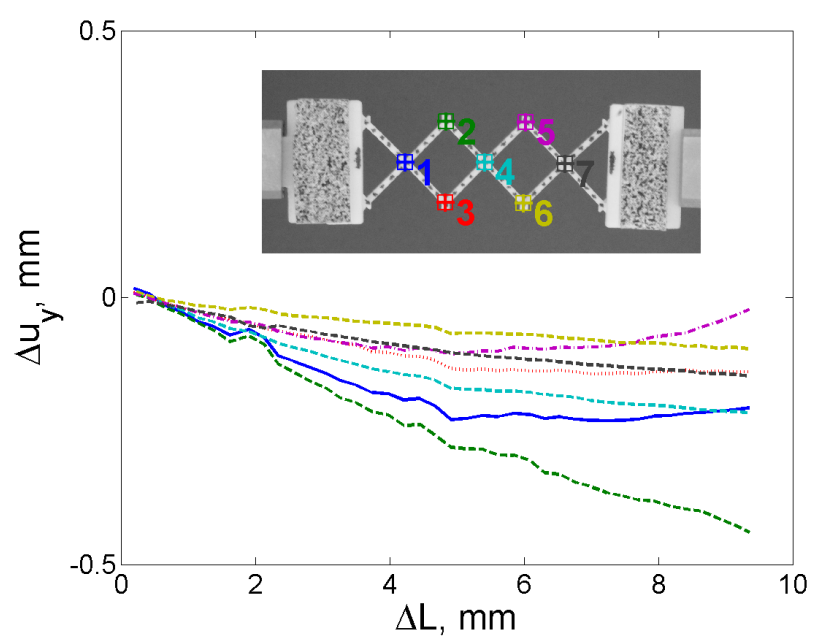

(b)

Figure 16: DIC analyses at the microscale of the bias extension test. History of displacement jumps in the horizontal (a) and vertical (b) directions. The inset shows the location and labels of the 7 hinges.

Let us also note that on a more quantitative way the experimental amplitudes are lower than the predicted levels. This fact can be explained by considering that (i) at the current state of research the fiber connectivity stiffness $\mathbb{K}_{c}$ and the constant $\mathfrak{k}_{3}$ were calibrated together with $\mathbb{K}_{p}$ and the parameter $\beta$ for obtaining the best fit of the experimental force-displacement curve. The predicted deformed shapes were consistent with experimental pictures. (ii) DIC analyses were performed on 2D pictures of exper-632 imental tests that were not totally in-plane. (iii) Last, the experimental conditions (e.g. 3D printed specimen with ${ }^{63}$ imperfections, clamping) are different from the ideal ones, ${ }_{635}^{634}$ which were assumed in the numerical simulations.

Figure 17 shows the displacement jumps for the bias ${ }^{636}$ compression test. For the vertical component (Fig- ${ }^{63}$ ure $17(b))$, the general trends are close to those obtained by numerical simulation (Figure 15). However, the lev- ${ }^{639}$ els are higher and nonlinearities are observed for the two extreme hinges ( 1 and 7 ). For the horizontal component of the displacement jumps (Figures 14 and 16(a)), the order of magnitude is closer even though more complex responses are observed in comparison with the simulations. It is believed that such differences point toward imperfections that induce 3D effects that were not accounted for in the $2 \mathrm{D}$ numerical simulations.

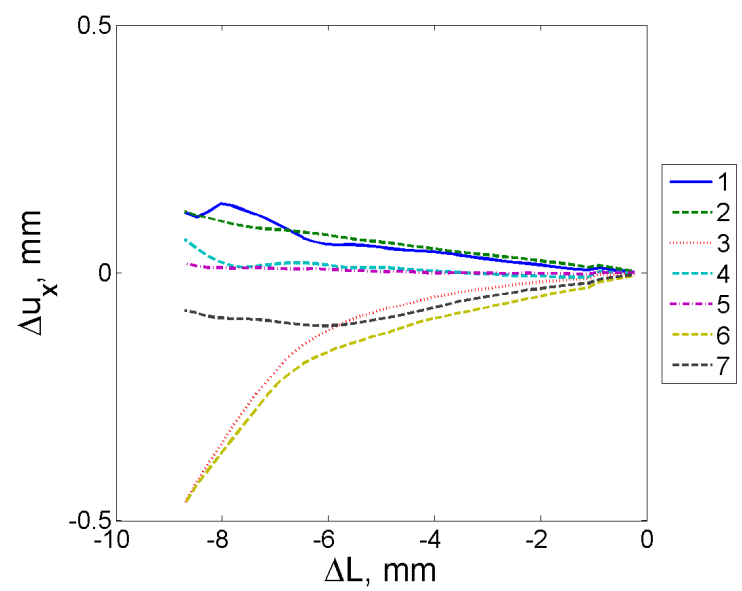

(a)

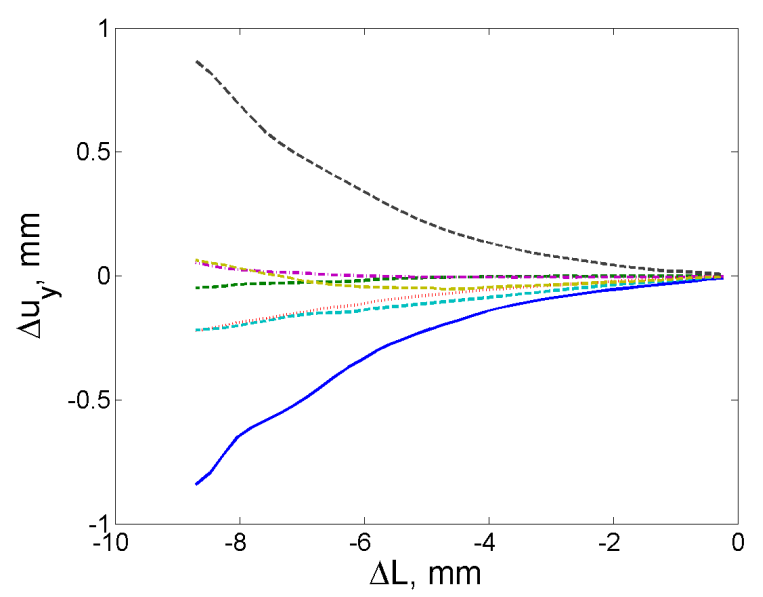

(b)

Figure 17: DIC analyses at the microscale of the bias compression test. The location and labels of the 7 hinges are identical to those of Figure 16 History of displacement jumps in the horizontal (a) and vertical (b) directions.

\section{Conclusion}

A model of a pantographic structure composed of nonlinear Euler-Bernoulli beams has been considered in this study. In this study the focus is on the mechanical behavior of hinges, which play a dramatically important role in the mechanics of pantographic structures. For this reason, we have chosen to study a structure consisting of only three cells, which makes it easier to focus on the hinges. As pointed out in the text, there is an asymmetry between 
the extension and compression tests. This asymmetry is698 related to the shear deformation of the pivots, which de-69s form differently and not symmetrically in the two tests:700 this result would be difficult to detect in the case of struc-701 tures with many beams and many pivots. In the presentro2 paper, a more in-depth study of the deformation mecha-703 nisms of pivots is presented. The energy term denoted as 704 fibre-connectivity was in fact only mentioned in previous705 works. Analyses conducted with the Digital Image Corre-700 lation technique allowed us to detect, in a qualitative way 707 at the current state of research, many details of the pivot7o8 deformations, thus providing an indication of the way forward in the modeling process.

The effect of hinge deformation on the mechanical re- ${ }^{700}$ sponse of pantographic cells was studied herein. As said, ${ }_{710}$ the model adopted for the analysis of such system was $_{711}$ based on the use of nonlinear Euler-Bernoulli beams. $A_{712}$ very important role is assumed by the shear deformation ${ }_{713}$ of individual hinges. From a macroscopic point of view, ${ }_{714}$ that deformation corresponds to relative motions between $n_{715}$ the beams of the two families that constitute the metama- ${ }_{716}$ terial. From a numerical point of view, the simulation of bias extension and compression tests would be symmetric ${ }_{718}$ in the absence of fiber connectivity deformation. However, ${ }_{719}$ this symmetry is lost thanks to fiber connectivity. From a purely experimental point of view, it was observed that the reaction forces measured in both the tests are not anti- ${ }^{720}$ symmetric but almost 5 times higher in magnitude in the bias extension test than in compression.

The model developed herein was calibrated with $\mathrm{a}^{722}$ unique set of parameters that could accurately describe ${ }^{723}$ the macroscopic load/displacement response of the stud- ${ }^{724}$ ied cells. Further, the deformed shapes were in qualitative ${ }^{725}$ agreement with the experimental observations. Since im- ${ }^{726}$ ages were acquired during the experiments studied herein, ${ }^{727}$ displacements could be measured via DIC. In the present ${ }^{728}$ case, relative motions at hinge ends could be measured ${ }^{729}$ thanks to a specially designed kinematic basis [19, which led to very low registration residuals. Displacement jumps730 could be measured and quantified for both tests. Some of the general trends predicted by the calibrated model were ${ }_{732}^{731}$ confirmed by the DIC measurements. Conversely, loss of $7_{733}^{32}$ symmetries points toward printing and experimental im-734 perfections of such centimetric objects.

The results obtained in this work deserve to be further ${ }_{737}^{736}$ investigated by means of other numerical tools. In par- ${ }_{738}$ ticular, the calibration of the model parameters may be ${ }^{739}$ performed at the hinge scale when based upon the kine- ${ }^{-70}$ matic measurements reported herein. This new route may ${ }_{742}^{741}$ lead to a better description of the hinge response that was w $_{743}$ not used for the calibration performed herein. One ques-744 tion that will then arise is whether all the hinges have the ${ }_{745}^{745}$ same overall response given the printing imperfections. $\mathrm{We}_{747}^{746}$ recall that a model similar to the one developed in [2] and 748 developed here was proposed in 6]. Suitable numerical ${ }^{749}$ algorithms, already available in the literature [7 9,18 , can be implemented for the detailed analysis of problems similar to the one we discussed.

As shown in Ref. 26, fiber connectivity induces failure modes that were not previously observed in pantographic structures. This observation and, in general, the study of damage in pantographic structures have motivated a recent study on the optimization of such structures [13. Various studies on damage in materials described by generalized models may be useful to refine the approach to damage in the specific case of pantographic structures. Some results available in the literature can be found in 23 .

\section{Authors' contributions}

M. Spagnuolo, U. Andreaus: Conceptualization, Methodology, Software.

M. Spagnuolo: Data curation, Writing, Original draft preparation.

A. Misra: Visualization, Investigation.

I. Giorgio, F. Hild: Supervision.

M. Spagnuolo, F. Hild: Software, Validation.

M. Spagnuolo, F. Hild, I. Giorgio: Writing, Reviewing and Editing.

\section{Acknowledgments}

Mario

Spagnuolo was supported by P.O.R. SARDEGNA F.S.E. 2014-2020 - Asse III "Istruzione e Formazione, Obiettivo Tematico: 10, Obiettivo Specifico: 10.5, Azione dell'accordo di Partenariato:10.5.12" Avviso di chiamata per il finanziamento di Progetti di ricerca - Anno 2017.

The authors want to thank Prof. T. Lekszycki (Warsaw University of Technology, Warsaw, Poland) for the production of the $3 \mathrm{D}$ printed specimens.

\section{References}

[1] Jean-Jacques Alibert, Pierre Seppecher, and Francesco dell'Isola. Truss modular beams with deformation energy depending on higher displacement gradients. Mathematics and Mechanics of Solids, 8(1):51-73, 2003.

[2] Ugo Andreaus, Mario Spagnuolo, Tomasz Lekszycki, and Simon R Eugster. A Ritz approach for the static analysis of planar pantographic structures modeled with nonlinear EulerBernoulli beams. Continuum Mechanics and Thermodynamics, pages $1-21,2018$.

[3] Emilio Barchiesi, Mario Spagnuolo, and Luca Placidi. Mechanical metamaterials: a state of the art. Mathematics and $\mathrm{Me}$ chanics of Solids, page 1081286517735695, 2018.

[4] Emilio Barchiesi, François Hild, and Francesco dell'Isola. On the Validation of Homogenized Modeling for Bi-Pantographic Metamaterials via Digital Image Correlation. International Journal of Solids and Structures, In press.(hal-02954418), 2020.

[5] Claude Boutin. Microstructural effects in elastic composites. International Journal of Solids and Structures, 33(7):1023-1051, 1996 
[6] Giuseppe Capobianco, Simon R Eugster, and Tom Winandy.82 Modeling planar pantographic sheets using a nonlinear Euler-822 Bernoulli beam element based on B-spline functions. PAMM,823 18(1):e201800220, 2018.

[7] Antonio Cazzani, Marcello Malagù, and Emilio Turco. Isogeo-825 metric analysis of plane-curved beams. Mathematics and Me-826 chanics of Solids, 21(5):562-577, 2016.

[8] Antonio Cazzani, Flavio Stochino, and Emilio Turco. An an-828 alytical assessment of finite element and isogeometric analyses 829 of the whole spectrum of timoshenko beams. ZAMM-Journals30 of Applied Mathematics and Mechanics/Zeitschrift für Ange-831 wandte Mathematik und Mechanik, 96(10):1220-1244, 2016.

[9] Antonio Cazzani, Mauro Serra, Flavio Stochino, and Emilios3 Turco. A refined assumed strain finite element model for statics834 and dynamics of laminated plates. Continuum Mechanics and835 Thermodynamics, 32:665-692, 2020.

10] Corentin Coulais, Chris Kettenis, and Martin van Hecke. A837 characteristic length scale causes anomalous size effects and838 boundary programmability in mechanical metamaterials. Na-839 ture Physics, 14(1):40-44, 2018

11] F dell'Isola, G Maier, U Perego, U Andreaus, R Esposito, and841 $\mathrm{S}$ Forest, editors. The complete works of Gabrio Piola: Volumes42 I (Advanced Structured Materials, vol. 38). Springer Verlag,843 Berlin (Germany), 2014.

[12] F dell'Isola, I Giorgio, Marek Pawlikowski, and NL Rizzi. Larges45 deformations of planar extensible beams and pantographic lat-846 tices: heuristic homogenization, experimental and numerical examples of equilibrium. Proc. R. Soc. A, 472(2185):20150790, 2016.

[13] Boris Desmorat, Mario Spagnuolo, and Emilio Turco. Stiffness optimization in nonlinear pantographic structures. Mathematics and Mechanics of Solids, DOI: 10.1177/1081286520935503, 2020.

[14] Victor A Eremeyev and Francesco dell'Isola. A note on reduced strain gradient elasticity. In Generalized Models and Non-classical Approaches in Complex Materials 1, pages 301310. Springer, 2018.

[15] Victor A Eremeyev, Giuseppe Rosi, and Salah Naili. Comparison of anti-plane surface waves in strain-gradient materials and materials with surface stresses. Mathematics and mechanics of solids, 24(8):2526-2535, 2019.

16] Paul Germain. The method of virtual power in continuum mechanics. part 2: Microstructure. SIAM Journal on Applied Mathematics, 25(3):556-575, 1973.

[17] Ivan Giorgio, Francesco dell'Isola, and Anil Misra. Chirality in 2D Cosserat Media Related to Stretch-Micro-rotation coupling with links to Granular Micromechanics. International Journal of Solids and Structures, 2020.

[18] L Greco, M Cuomo, and L Contrafatto. A quadrilateral g1conforming finite element for the kirchhoff plate model. Computer Methods in Applied Mechanics and Engineering, 346:913951, 2019.

[19] François Hild, A Misra, and F dell'Isola. Multiscale DIC applied to Pantographic Structures. Experimental Mechanics, pages 113, 2020.

[20] Florent Mathieu, Hugo Leclerc, François Hild, and Stéphane Roux. Estimation of elastoplastic parameters via weighted femu and integrated-dic. Experimental Mechanics, 55(1):105-119, 2015.

[21] Raymond David Mindlin. Microstructure in linear elasticity. Technical report, Columbia Univ New York Dept of Civil Engineering and Engineering Mechanics, 1963.

[22] N Nejadsadeghi, M De Angelo, R Drobnicki, T Lekszycki, $\mathrm{F}$ dell'Isola, and A Misra. Parametric experimentation on pantographic unit cells reveals local extremum configuration. Experimental Mechanics, 59(6):927-939, 2019.

[23] Luca Placidi and Emilio Barchiesi. Energy approach to brittle fracture in strain-gradient modelling. Proc. R. Soc. A, 474 (2210):20170878, 2018.

[24] Daria Scerrato, Ivan Giorgio, and Nicola Luigi Rizzi. Threedimensional instabilities of pantographic sheets with parabolic lattices: numerical investigations. Zeitschrift für angewandte Mathematik und Physik, 67(3):53, 2016.

[25] RV Southwell. XVI. On Castigliano's theorem of least work, and the principle of St. Venant. The London, Edinburgh, and Dublin Philosophical Magazine and Journal of Science, 45(265): 193-212, 1923.

[26] M Spagnuolo, K Barcz, A Pfaff, F dell'Isola, and P Franciosi. Qualitative pivot damage analysis in aluminum printed pantographic sheets: Numerics and experiments. Mechanics Research Communications, 83:47-52, 2017.

[27] Mario Spagnuolo, Patrice Peyre, and Corinne Dupuy. Phenomenological aspects of quasi-perfect pivots in metallic pantographic structures. Mechanics Research Communications, 101: 103415, 2019.

[28] Michael A Sutton. Computer vision-based, noncontacting deformation measurements in mechanics: a generational transformation. Applied Mechanics Reviews, 65(5), 2013.

[29] M Erden Yildizdag, Emilio Barchiesi, and Francesco dell'Isola. Three-point bending test of pantographic blocks: numerical and experimental investigation. Mathematics and Mechanics of Solids, page 1081286520916911, 2020.

[30] Hong Zhang, Xiaoyun Xie, Yiwei Xie, CM Wang, and Pengcheng Jiao. Extensible beam-like metastructures at the microscale: Theoretical and modified hencky bar-chain modeling. International Journal of Mechanical Sciences, 180:105636, 2020 